Article

\title{
An Interference Cancellation Scheme for High Reliability Based on MIMO Systems
}

\author{
Jae-Hyun Ro, Sung-Soon Park and Hyoung-Kyu Song * \\ uT Communication Research Institute, Sejong University, Gunja-dong 98, Gwangjin-gu, Seoul 05006, Korea; \\ ilovebisu@nate.com (J.-H.R.); sspark9207@naver.com (S.-S.P.) \\ * Correspondence: songhk@sejong.ac.kr; Tel.: +82-2-3408-3890
}

Received: 9 February 2018; Accepted: 16 March 2018; Published: 18 March 2018

\begin{abstract}
This article proposes a new interference cancellation scheme in a half-duplex based two-path relay system. In the conventional two-path relay system, inter-relay-interference (IRI) which severely degrades the error performances at a destination occurs because a source and a relay transmit signals simultaneously at a specific time. The proposed scheme removes the IRI at a relay for higher signal-to-interference plus noise ratio (SINR) to receive interference free signal at a destination, unlike the conventional relay system, which removes IRI at a destination. To handle the IRI, the proposed scheme uses multiple-input multiple-output (MIMO) signal detection at the relays and it makes low-complexity signal processing at a destination which is a usually mobile user. At the relays, the proposed scheme uses the low-complexity QR decomposition-M algorithm (QRD-M) to optimally remove the IRI. Also, for obtaining diversity gain, the proposed scheme uses cyclic delay diversity (CDD) to transmit the signals at a source and the relays. In simulation results, the error performance for the proposed scheme is better when the distance between one relay and another relay is low unlike the conventional scheme because the QRD- $M$ detects received signal in order of higher post signal-to-noise ratio (SNR).
\end{abstract}

Keywords: IRI; relay; CDD; QRD-M

\section{Introduction}

Due to an increasing amount of traffic in recent wireless communication systems, many schemes for high reliability and bandwidth efficiency have been studied. One of the systems to obtain high data rate and bandwidth efficiency is orthogonal frequency division multiplexing (OFDM) [1,2]. Also, relay systems such as amplifying-and-forward (AF) and decoding-and-forward (DF) have been studied for expanding cell coverage [3]. For practical realization of the relay systems, a half-duplex relay is usually used. Among half-duplex based relay systems, the cooperative communication system is very popular. The cooperative communication system, which is a virtual multiple input multiple output (MIMO) system obtains high diversity order with combined gain at a destination due to independent path of channel state from a source to a destination and a relay to a destination [4,5]. However, the half-duplex based relay cannot transmit and receive signals at the same time and it results in the degradation of channel capacity compared to direct point-to-point communication systems. So, general relaying system such as the cooperative communication system requires many time slots to transmit several data streams and it causes low data rate for overall systems. To solve this problem, two-path relay scheme is considered in this article. In the two-path relay system, there are one source node, one destination node and two half-duplex relay nodes. In an odd time slot or an even time slot, a source transmits signals alternately to the relay nodes. Since a source can transmit signal to the relays continuously, the main problem for degradation of channel capacity in a single relay is resolved. However, simultaneous transmissions from a source to a relay and from one relay to another relay 
cause interferences at the relays and these interferences are called as inter-relay interference (IRI) [6-8]. The IRI severely degrades overall signal-to-interference plus noise ratio (SINR) at a receiver and it makes very hard to decode the received signals at a destination. In [6], full interference cancellation (FIC) is used to remove the IRI at a destination. When the number of transmit data streams is $N$, $(N+1)$ time slots are required and resultant data rate for the FIC is $N /(N+1)$ which is close to the full data rate of 1 and has very high bandwidth efficiency. When $N$ is very large, the resultant data rate for the FIC is approximate to 1 . However, the error rate for the conventional FIC is very high because the relays transmit signals which are suffer from the IRI and it causes severe error propagations. Finally, the error performance for the conventional FIC is degraded when a distance between one relay and another relay decreases because the effects of interferences increase and are propagated to a destination.

This article proposes a new interference cancellation scheme for high reliability in half-duplex based two-path relay system. Unlike the conventional FIC, the proposed scheme completely cancels the IRI at relay nodes and the effects of error propagations are low at a destination. To remove the IRI at relay nodes, the proposed scheme uses MIMO signal detection [9-18]. The complexity for the MIMO signal detection is high at a receiver. However, complex signal detection may be applied well when the receiver is a relay which is not usually mobile user. To optimally cancel the IRI, the relays use the low-complexity QR decomposition- $M$ algorithm (QRD-M) in [13] which has the same error performance as maximum likelihood (ML). The conventional QRD-M is reduced complexity version of the ML. However, its complexity is very high as the used modulation order, the number of transmit antennas is increased. As shown in simulation results in Section 5, the used modulation scheme is 256-quadrature amplitude modulation (QAM). So, the low-complexity QRD-M is used. The main reason for high complexity of the conventional QRD- $M$ is that it calculates many unnecessary survival paths at all layers. To reduce the number of unnecessary survival paths, [13] eliminates unnecessary survival paths which have larger accumulated squared Euclidean distance (ASED) than adaptively calculated threshold at each layer.

\section{Conventional Two-Path Relay System Model}

The conventional two-path relay system is shown in Figure 1. $\mathrm{S}$ denotes a source, $\mathrm{R}_{1}$ and $\mathrm{R}_{2}$ denotes half-duplex relays and D denotes a destination. $h_{\mathrm{SR}_{1}}$ and $h_{\mathrm{SR}_{2}}$ are channel coefficients from $S$ to $R_{1}$ and $S$ to $R_{2}$, respectively. $h_{R_{1} D}$ and $h_{R_{2} D}$ are channel coefficients from $R_{1}$ to $D$ and $R_{2}$ to $D$, respectively. $h_{R_{1} R_{2}}$ and $h_{R_{2} R_{1}}$ are inter-relay channel coefficients from $R_{1}$ to $R_{2}$ and $R_{2}$ to $R_{1}$, respectively. The relays forward received signals from $S$ by using the AF scheme. In an odd time slot, $S$ transmits symbol to $R_{1}$ and the other relay $R_{2}$ relays the symbol which is received from $S$ at previous time slot. Likewise, in an even time slot, $S$ transmits symbol to $R_{2}$ and the other relay $R_{1}$ relays the symbol which is received from $S$. In the two-path relay system, since $S$ and one of the relay nodes transmit symbol simultaneously in a specific time slot, IRI occur at relay nodes. Table 1 shows configuration for transmission symbols of conventional scheme. According to transmit symbol for each time slot in Table 1 , the received symbol at $\mathrm{R}_{1}$ for time slot $n$ is as follows,

$$
y_{\mathrm{R}_{1}}(n)=h_{\mathrm{SR}_{1}} x_{\mathrm{S}}(n)+h_{\mathrm{R}_{1} \mathrm{R}_{2}} x_{\mathrm{R}_{2}}(n)+z_{\mathrm{R}_{1}}(n),
$$

where $x_{\mathrm{S}}$ is transmit symbol from $\mathrm{S}, x_{\mathrm{R}_{2}}$ is transmit symbol from $\mathrm{R}_{2}, z_{\mathrm{R}_{1}}$ is zero-mean additive white Gaussian noise (AWGN) at $\mathrm{R}_{1}$ and $n$ is assumed to be odd number. 


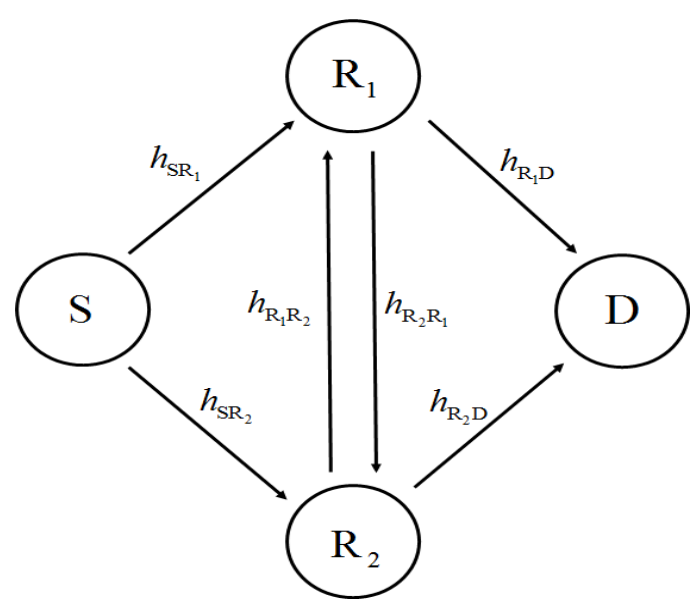

Figure 1. The conventional two-path relay system.

Table 1. The configuration for transmission symbols of conventional scheme.

\begin{tabular}{cccc}
\hline Time Slot & $\mathbf{S}$ & $\mathbf{R}_{\mathbf{1}}$ & $\mathbf{R}_{\mathbf{2}}$ \\
\hline$t=1$ & $\begin{array}{c}x_{\mathrm{S}}(1) \\
\left(\mathrm{S} \rightarrow \mathrm{R}_{1}\right)\end{array}$ & - & - \\
\hline$t=2$ & $\begin{array}{c}x_{\mathrm{S}}(2) \\
\left(\mathrm{S} \rightarrow \mathrm{R}_{2}\right)\end{array}$ & $\begin{array}{c}\beta_{1} y_{\mathrm{R}_{1}}(1) \\
\left(\mathrm{R}_{1} \rightarrow \mathrm{D}, \mathrm{R}_{1} \rightarrow \mathrm{R}_{2}\right)\end{array}$ & - \\
\hline$t=3$ & $\begin{array}{c}x_{\mathrm{S}}(3) \\
\left(\mathrm{S} \rightarrow \mathrm{R}_{1}\right)\end{array}$ & - & $\beta_{2} y_{\mathrm{R}_{2}}(2)$ \\
& $\vdots$ & $\vdots$ & $\left(\mathrm{R}_{2} \rightarrow \mathrm{D}, \mathrm{R}_{2} \rightarrow \mathrm{R}_{1}\right)$ \\
\hline$\vdots$ & $\begin{array}{c}x_{\mathrm{S}}(n) \\
\left(\mathrm{S} \rightarrow \mathrm{R}_{1}\right)\end{array}$ & - & $\vdots$ \\
\hline \begin{tabular}{c} 
Odd $n)$ \\
\hline
\end{tabular} & & $\beta_{n-1} y_{\mathrm{R}_{2}}(n-1)$ \\
$\left(\mathrm{R}_{2} \rightarrow \mathrm{D}_{2} \rightarrow \mathrm{R}_{1}\right)$
\end{tabular}

In Equation (1), the second term of the right-hand side is interference. In the next time slot, $R_{1}$ relays the received symbol to $\mathrm{D}$ using the $\mathrm{AF}$ and the received symbol at $\mathrm{D}$ is as follows,

$$
\begin{aligned}
y_{\mathrm{D}}(n+1) & =\beta_{1} y_{\mathrm{R}_{1}}(n) h_{\mathrm{R}_{1} \mathrm{D}}+z_{\mathrm{D}}(n+1), \\
& =\beta_{1}\left(h_{\mathrm{SR}_{1}} x_{\mathrm{S}}(n)+h_{\mathrm{R}_{1} \mathrm{R}_{2}} x_{\mathrm{R}_{2}}(n)\right)+\widetilde{z}_{\mathrm{D}}(n+1),
\end{aligned}
$$

where $z_{\mathrm{D}}$ is zero-mean AWGN at $\mathrm{D}, \widetilde{z}_{\mathrm{D}}=z_{D}+\beta_{1} z_{R_{1}}$ is new zero-mean AWGN and $\beta_{1}$ is an amplifying factor as follows,

$$
\beta_{1}=\sqrt{\frac{P_{\mathrm{R}_{1}}}{\left|h_{\mathrm{SR}_{1}}\right|^{2} P_{\mathrm{S}}+\left|h_{\mathrm{R}_{1} \mathrm{R}_{2}}\right|^{2} P_{\mathrm{R}_{2}}+\sigma^{2}}}
$$

where $P_{S}, P_{R_{1}}$ and $P_{R_{2}}$ are transmit power at $S, R_{1}$ and $R_{2}$, respectively, and $\sigma^{2}$ is noise power.

Substituting Equation (1) into (2), the received symbol at D is as follows,

$$
y_{\mathrm{D}}(n+1)=\beta_{1} x_{\mathrm{S}}(n) h_{\mathrm{SR}_{1}} h_{\mathrm{R}_{1} \mathrm{D}}+\beta_{1} x_{\mathrm{R}_{2}}(n) h_{\mathrm{R}_{1} \mathrm{R}_{2}} h_{\mathrm{R}_{1} \mathrm{D}}+\beta_{1} h_{\mathrm{R}_{1} \mathrm{D}} z_{\mathrm{R}_{1}}(n)+z_{\mathrm{D}}(n+1) .
$$

Because $\mathrm{R}_{1}$ relays the symbol to $\mathrm{D}$ at time slot $n, y_{\mathrm{D}}(n)$ is given by,

$$
y_{\mathrm{D}}(n)=x_{\mathrm{R}_{2}}(n) h_{\mathrm{R}_{2} \mathrm{D}}+z_{\mathrm{D}}(n),
$$

or Equation (5) is written as follows, 


$$
x_{\mathrm{R}_{2}}(n)=\frac{y_{\mathrm{D}}(n)-z_{\mathrm{D}}(n)}{h_{\mathrm{R}_{2} \mathrm{D}}} .
$$

Substituting Equation (6) into (4), $y_{\mathrm{D}}(n+1)$ is as follows,

$$
y_{\mathrm{D}}(n+1)=\beta_{1} x_{\mathrm{S}}(n) h_{\mathrm{SR}_{1}} h_{\mathrm{R}_{1} \mathrm{D}}+\frac{\beta_{1} h_{\mathrm{R}_{1} \mathrm{R}_{2}} h_{\mathrm{R}_{1} \mathrm{D}}}{h_{\mathrm{R}_{2} \mathrm{D}}} y_{\mathrm{D}}(n)+\widetilde{z}(n),
$$

where $\widetilde{z}(n)$ is a new noise as follows,

$$
\widetilde{z}(n)=\beta_{1} h_{\mathrm{R}_{1} \mathrm{D}} z_{\mathrm{R}_{1}}(n)+z_{\mathrm{D}}(n+1)-\frac{\beta_{1} h_{\mathrm{R}_{1} \mathrm{R}_{2}} h_{\mathrm{R}_{1} \mathrm{D}}}{h_{\mathrm{R}_{2} \mathrm{D}}} z_{\mathrm{D}}(n) .
$$

In Equation (7), the second term of the right-hand side is IRI and it is cancelled at $\mathrm{D}$ by subtracting $\frac{\beta_{1} h_{R_{1} R_{2}} h_{R_{1} \mathrm{D}}}{h_{R_{2} \mathrm{D}}} y_{\mathrm{D}}(n)[6]$. Finally, IRI free symbol $\widetilde{y}_{\mathrm{D}}$ is as follows,

$$
\widetilde{y}_{\mathrm{D}}(n+1)=\beta_{1} x_{\mathrm{s}}(n) h_{\mathrm{SR}_{1}} h_{\mathrm{R}_{1} \mathrm{D}}+\widetilde{z}(n) .
$$

Similarly, if $n$ is an even number, the received symbol at D including IRI is as follows,

$$
y_{\mathrm{D}}(n+1)=\beta_{2} x_{\mathrm{s}}(n) h_{\mathrm{SR}_{2}} h_{\mathrm{R}_{2} \mathrm{D}}+\frac{\beta_{2} h_{\mathrm{R}_{2} \mathrm{R}_{1}} h_{\mathrm{R}_{2} \mathrm{D}}}{h_{\mathrm{R}_{1} \mathrm{D}}} y_{\mathrm{D}}(n)+\widetilde{z}(n),
$$

where $\beta_{2}$ is amplifying factor as follows,

$$
\beta_{2}=\sqrt{\frac{P_{\mathrm{R}_{2}}}{\left|h_{\mathrm{SR}_{2}}\right|^{2} P_{\mathrm{S}}+\left|h_{\mathrm{R}_{2} \mathrm{R}_{1}}\right|^{2} P_{\mathrm{R}_{1}}+\sigma^{2}}} .
$$

The conventional scheme removes IRI at a destination by subtracting IRI from the received symbol. However, the error performance is decreased severely when two relay nodes are close to each other because the effects of interference are increased and are propagated to a destination node. Also, the conventional two-path relay system removes interferences not at a relay but a destination. So, when the conventional two-path relay system uses the DF scheme at a relay, it has high probability that the regenerated signals at a relay have many errors and it causes severe error propagations at a destination. So, the proposed scheme uses MIMO signal detection at the relays to remove IRI at the relays (not at a destination) and the DF scheme can be used at the relays due to IRI free symbol. For removal of IRI, the proposed scheme uses low-complexity QRD- $M$ which has optimal error performance and very lower complexity than the conventional QRD-M. Finally, the source and the relays use cyclic delay diversity (CDD) [19] which is simple spatial diversity scheme to obtain spatial diversity gain and higher error performance.

\section{Proposed Scheme}

The proposed two-path relay system is shown in Figure 2. All nodes have two antennas for simple representation. Also, DF method is used at $R_{1}$ and $R_{2}$ to relay the received symbol from $S$. In the proposed scheme, $S$ transmits symbols by using CDD and $R_{1}$ and $R_{2}$ also use CDD after decoding and re-encoding the received symbols from $\mathrm{S}$ for obtaining spatial diversity gain. The CDD is spatial diversity scheme which is easily used in MIMO-OFDM systems because transmit symbols are cyclically delayed version for the original transmit symbol. Due to the cyclic shift, the frequency selectivity of channel is increased and the error distribution is changed. So, the error performance can be improved when the channel coding scheme is used because the error is distributed at the receiver [19]. In $N_{t} \times N_{r}$ MIMO system where $N_{t}$ and $N_{r}$ are the number of transmit and receive antennas, the received symbol $\mathbf{Y}=\left[\begin{array}{llll}y_{1} & y_{2} & \cdots & y_{N_{r}}\end{array}\right]^{T}$ is as follows, 


$$
\left[\begin{array}{c}
y_{1} \\
y_{2} \\
\vdots \\
y_{N_{r}}
\end{array}\right]=\left[\begin{array}{cccc}
h_{11} & h_{12} & \cdots & h_{1 N_{t}} \\
h_{21} & h_{11} & \cdots & h_{2 N_{t}} \\
\vdots & \vdots & \ddots & \vdots \\
h_{N_{r} 1} & h_{N_{r} 2} & \cdots & h_{N_{r} N_{t}}
\end{array}\right]\left[\begin{array}{c}
x_{1} \\
x_{2} \\
\vdots \\
x_{N_{t}}
\end{array}\right]+\left[\begin{array}{c}
z_{1} \\
z_{2} \\
\vdots \\
z_{N_{r}}
\end{array}\right],
$$

where $h_{i j}\left(i=1,2, \cdots, N_{r}, j=1,2, \cdots, N_{t}\right)$ is a channel coefficient from the $j$-th transmit antenna to the $i$-th receive antenna, $x_{j}$ is transmit symbol from the $j$-th transmit antenna which is $\delta_{j}$ cyclically delayed symbol for the original transmit symbol $x$ and $z_{i}$ is a zero-mean AWGN at the $i$-th receive antenna.

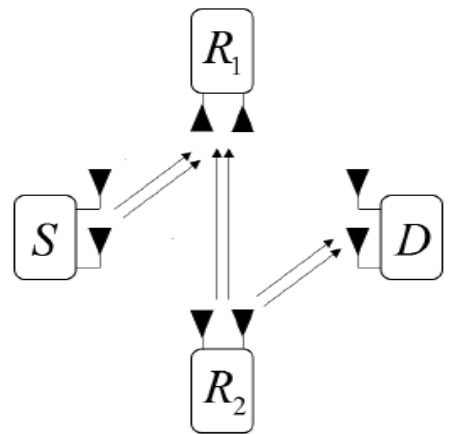

(a)

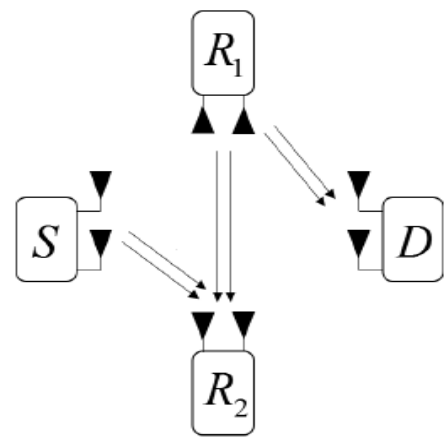

(b)

Figure 2. The proposed two-path relay MIMO system. (a) Odd time slot; (b) Even time slot. MIMO: multiple input multiple output.

In the CDD, the relationship for two different transmit symbols between $x_{m}$ and $x_{n}$ is as follows,

$$
x_{m}=e^{-j \frac{2 \pi k\left(\delta_{n}-\delta_{m}\right)}{N}} x_{n}
$$

where $N$ is fast Fourier transform (FFT) size.

Substituting Equation (13) into (12) when it is assumed that the cyclic delay at the first antenna $\delta_{1}$ is zero for simple representations, Equation (12) is rewritten as follows,

$$
\left[\begin{array}{c}
y_{1} \\
y_{2} \\
\vdots \\
y_{N_{r}}
\end{array}\right]=\left[\begin{array}{c}
h_{\mathrm{C}, 1} \\
h_{\mathrm{C}, 2} \\
\vdots \\
h_{\mathrm{C}, N_{r}}
\end{array}\right] x+\left[\begin{array}{c}
z_{1} \\
z_{2} \\
\vdots \\
z_{N_{r}}
\end{array}\right]
$$

where $h_{\mathrm{C}, i}=\sum_{j=1}^{N_{t}} h_{i j} e^{-j \frac{2 \pi k \delta_{j}}{N}}$ is composite channel due to property of Equation (13) and $x$ is non-cyclic delayed original transmit symbol.

Finally, transmit symbols are estimated by using maximum ratio combining (MRC) [20] in Equation (14) as follows,

$$
x=\underbrace{\sum_{i=1}^{N_{r}} h_{\mathrm{C}, i}^{H} y_{i}}_{(\mathrm{a})} / \underbrace{\sum_{i=1}^{N_{r}}\left|h_{\mathrm{C}, i}\right|^{2}}_{(\mathrm{b})} .
$$

Table 2 shows configuration for the transmit symbols of the proposed scheme. It is assumed that $n$ is an odd number. $S$ transmits a symbol to $R_{1}$ in an odd time slot and to $R_{2}$ in an even time slot. For the transmit symbols in Table 2, the received symbols without noise at $R_{1}$ for an odd $n$ except for 1 are as follows, 


$$
\left[\begin{array}{l}
y_{\mathrm{R}_{1}, 1}(n) \\
y_{\mathrm{R}_{1}, 2}(n)
\end{array}\right]=\underbrace{\left[\begin{array}{ll}
h_{\mathrm{SR}_{1}, 11} & h_{\mathrm{SR}_{1}, 12} \\
h_{\mathrm{SR}_{1}, 21} & h_{\mathrm{SR}_{1}, 22}
\end{array}\right]\left[\begin{array}{l}
x_{\delta_{1}}(n) \\
x_{\delta_{2}}(n)
\end{array}\right]}_{\text {Desired }}+\underbrace{\left[\begin{array}{ll}
h_{\mathrm{R}_{2} \mathrm{R}_{1}, 11} & h_{\mathrm{R}_{2} \mathrm{R}_{1}, 12} \\
h_{\mathrm{R}_{2} \mathrm{R}_{1}, 21} & h_{\mathrm{R}_{2} \mathrm{R}_{1}, 22}
\end{array}\right]\left[\begin{array}{l}
\hat{x}_{\delta_{1}}(n-1) \\
\hat{x}_{\delta_{2}}(n-1)
\end{array}\right]}_{\text {IRI }}
$$

where $h_{\mathrm{SR}_{1}, i j}$ is a channel coefficient from the $j$-th transmit antenna at $S$ to the $i$-th receive antenna at $\mathrm{R}_{1}$ and $h_{\mathrm{R}_{2} \mathrm{R}_{1}, i j}$ is a channel coefficient from the $j$-th transmit antenna at $\mathrm{R}_{2}$ to the $i$-th receive antenna at $\mathrm{R}_{1}$.

Table 2. The configuration for transmission symbols of proposed scheme.

\begin{tabular}{|c|c|c|c|c|c|c|}
\hline \multirow[t]{2}{*}{ Time Slot } & \multicolumn{2}{|c|}{$S$} & \multicolumn{2}{|c|}{$\mathbf{R}_{\mathbf{1}}$} & \multicolumn{2}{|c|}{$\mathbf{R}_{\mathbf{2}}$} \\
\hline & $\mathrm{Tx}_{1}$ & $\mathrm{Tx}_{2}$ & $\mathrm{Tx}_{1}$ & $\mathrm{Tx}_{2}$ & $\mathrm{Tx}_{1}$ & $\mathrm{Tx}_{2}$ \\
\hline$t=1$ & $\begin{array}{c}x_{1} \\
\left(\mathrm{~S} \rightarrow \mathrm{R}_{1}\right)\end{array}$ & $\begin{array}{c}x_{1, \delta} \\
\left(\mathrm{S} \rightarrow \mathrm{R}_{1}\right)\end{array}$ & & & & \\
\hline$t=2$ & $\begin{array}{c}x_{2} \\
\left(\mathrm{~S} \rightarrow \mathrm{R}_{2}\right)\end{array}$ & $\begin{array}{c}x_{2, \delta} \\
\left(\mathrm{S} \rightarrow \mathrm{R}_{2}\right)\end{array}$ & $\begin{array}{c}\hat{x}_{1} \\
\left(\mathrm{R}_{1} \rightarrow \mathrm{D},\right. \\
\left.\mathrm{R}_{1} \rightarrow \mathrm{R}_{2}\right)\end{array}$ & $\begin{array}{c}\hat{x}_{1, \delta} \\
\left(\mathrm{R}_{1} \rightarrow \mathrm{D}\right. \\
\left.\mathrm{R}_{1} \rightarrow \mathrm{R}_{2}\right)\end{array}$ & & \\
\hline$t=3$ & $\begin{array}{c}x_{3} \\
\left(\mathrm{~S} \rightarrow \mathrm{R}_{1}\right)\end{array}$ & $\begin{array}{c}x_{3, \delta} \\
\left(\mathrm{S} \rightarrow \mathrm{R}_{1}\right)\end{array}$ & & & $\begin{array}{c}\hat{x}_{2} \\
\left(\mathrm{R}_{2} \rightarrow \mathrm{D},\right. \\
\left.\mathrm{R}_{2} \rightarrow \mathrm{R}_{1}\right)\end{array}$ & $\begin{array}{c}\hat{x}_{2, \delta} \\
\left(\mathrm{R}_{2} \rightarrow \mathrm{D}\right. \\
\left.\mathrm{R}_{2} \rightarrow \mathrm{R}_{1}\right)\end{array}$ \\
\hline$\vdots$ & $\vdots$ & $\vdots$ & $\vdots$ & $\vdots$ & $\vdots$ & $\vdots$ \\
\hline $\begin{array}{c}t=n \\
(\operatorname{Odd} n)\end{array}$ & $\begin{array}{c}x_{n} \\
\left(\mathrm{~S} \rightarrow \mathrm{R}_{1}\right)\end{array}$ & $\begin{array}{c}x_{n, \delta} \\
\left(\mathrm{S} \stackrel{\left.\mathrm{R}_{1}\right)}{ }\right)\end{array}$ & & & $\begin{array}{c}\hat{x}_{n-1} \\
\left(\mathrm{R}_{2} \rightarrow \mathrm{D}\right. \\
\left.\mathrm{R}_{2} \rightarrow \mathrm{R}_{1}\right)\end{array}$ & $\begin{array}{c}\hat{x}_{n-1, \delta} \\
\left(\mathrm{R}_{2} \rightarrow \mathrm{D},\right. \\
\left.\mathrm{R}_{2} \rightarrow \mathrm{R}_{1}\right)\end{array}$ \\
\hline
\end{tabular}

Using the property of Equation (13), Equation (16) is represented as follows,

$$
\left[\begin{array}{l}
y_{\mathrm{R}_{1}, 1}(n) \\
y_{\mathrm{R}_{1}, 2}(n)
\end{array}\right]=\left[\begin{array}{ll}
h_{\mathrm{SR}_{1}, 11}+e^{-j \frac{2 \pi k \delta_{2}}{N}} h_{\mathrm{SR}_{1}, 12} & h_{\mathrm{R}_{2} \mathrm{R}_{1}, 11}+e^{-j \frac{2 \pi k \delta_{2}}{N}} h_{\mathrm{R}_{2} \mathrm{R}_{1}, 12} \\
h_{\mathrm{SR}_{1}, 21}+e^{-j \frac{2 \pi k \delta_{2}}{N}} h_{\mathrm{SR}_{1}, 22} & h_{\mathrm{R}_{2} \mathrm{R}_{1}, 21}+e^{-j \frac{2 \pi k \delta_{2}}{N}} h_{\mathrm{R}_{2} \mathrm{R}_{1}, 12}
\end{array}\right]\left[\begin{array}{c}
\underbrace{x(n)}_{\text {Desired }} \\
\underbrace{\hat{x}(n-1)}_{\mathrm{IRI}}
\end{array}\right] .
$$

Likewise, received symbols at $\mathrm{R}_{2}$ for an even $n$ are as follows,

$$
\left[\begin{array}{l}
y_{\mathrm{R}_{2}, 1}(n) \\
y_{\mathrm{R}_{2}, 2}(n)
\end{array}\right]=\underbrace{\left[\begin{array}{ll}
h_{\mathrm{SR}_{2}, 11} & h_{\mathrm{SR}_{2}, 12} \\
h_{\mathrm{SR}_{2}, 21} & h_{\mathrm{SR}_{2}, 22}
\end{array}\right]\left[\begin{array}{l}
x_{\delta_{1}}(n) \\
x_{\delta_{2}}(n)
\end{array}\right]}_{\text {Desired }}+\underbrace{\left[\begin{array}{ll}
h_{\mathrm{R}_{1} \mathrm{R}_{2}, 11} & h_{\mathrm{R}_{1} \mathrm{R}_{2}, 12} \\
h_{\mathrm{R}_{1} \mathrm{R}_{2}, 21} & h_{\mathrm{R}_{1} \mathrm{R}_{2}, 22}
\end{array}\right]\left[\begin{array}{l}
\hat{x}_{\delta_{1}}(n+1) \\
\hat{x}_{\delta_{2}}(n+1)
\end{array}\right]}_{\text {IRI }}
$$

where $h_{\mathrm{SR}_{2}, i j}$ is a channel coefficient from the $j$-th transmit antenna at $S$ to the $i$-th receive antenna at $R_{2}$ and $h_{\mathrm{R}_{1} \mathrm{R}_{2}, i j}$ is a channel coefficient from the $j$-th transmit antenna at $\mathrm{R}_{1}$ to the $i$-th receive antenna at $\mathrm{R}_{2}$.

Like Equation (17), Equation (18) is represented as follows,

$$
\left[\begin{array}{l}
y_{\mathrm{R}_{2}, 1}(n) \\
y_{\mathrm{R} 2,2}(n)
\end{array}\right]=\left[\begin{array}{ll}
h_{\mathrm{SR}_{2}, 11}+e^{-j \frac{2 \pi k \delta_{2}}{N}} h_{\mathrm{SR}_{2}, 12} & h_{\mathrm{R}_{1} \mathrm{R}_{2}, 11}+e^{-j \frac{2 \pi k \delta_{2}}{N}} h_{\mathrm{R}_{1} \mathrm{R}_{2}, 12} \\
h_{\mathrm{SR}_{2}, 21}+e^{-j \frac{2 \pi k \delta_{2}}{N}} h_{\mathrm{SR}_{2}, 22} & h_{\mathrm{R}_{1} \mathrm{R}_{2}, 21}+e^{-j \frac{2 \pi k \delta_{2}}{N}} h_{\mathrm{R}_{1} \mathrm{R}_{2}, 22}
\end{array}\right]\left[\begin{array}{c}
\underbrace{x(n)}_{\text {Desired }} \\
\underbrace{\hat{x}(n+1)}_{\mathrm{IRI}}
\end{array}\right] .
$$

For obtaining desired symbols, the proposed scheme removes IRI at $R_{1}$ and $R_{2}$ using MIMO signal detection scheme. Among several signal detections, the proposed scheme uses low-complexity 
QRD- $M$ which has optimal error performance. The QRD- $M$ is reduced complexity of the ML. However, the complexity for QRD-M is increased exponentially as modulation order and the number of transmit antennas is large because unnecessary survival paths at each layer are also increased exponentially. In [13], thresholds are calculated at each layer to eliminate unnecessary survival paths. For path eliminations, ASED is compared with a calculated threshold at each layer and paths which are larger than a threshold are eliminated. After applying low-complexity QRD-M, IRI is fully removed. Then, $R_{1}$ and $R_{2}$ transmit decoded symbols by using CDD and D decodes received symbols by using MRC. Also, transmit symbols at other time slots are similarly processed.

\section{Simulation Results}

This section shows simulation results for bit error rate (BER) performance and complexity of the proposed scheme. The simulation parameters are shown in Table 3. Specifically, the loss of power for received symbol according to the distance from a node to a node is considered and it is represented as $L$ as follows,

$$
L=\sqrt{10^{P L / 10}},
$$

where $P L=-10 p \log _{10} d$ denotes path loss where $d$ is distance from a node to a node and $p$ is path loss exponent which is set to be 3 in this simulation.

Table 3. The simulation parameters.

\begin{tabular}{cc}
\hline Parameters & Scheme or Values \\
\hline FFT size & 128 \\
GI size & 32 \\
Modulation scheme & 256-QAM \\
Channel model & 8-path Rayleigh fading channel \\
Channel coding \& Constraint length & $1 / 2$ convolutional code \& 3 \\
2 for each node
\end{tabular}

Figure 3 shows BER performances with respect to SNR for the conventional FIC and the proposed scheme. The distances from a source to a relay, a relay to a destination, and one relay to another relay are normalized to 1 . Also, the BER performances which use the ML at a relay for IRI cancellation are also shown. The proposed scheme has very higher BER performance than the conventional FIC because a relay transmits symbol which does not include interference symbol from another relay. However, a relay of the conventional FIC transmits symbol which includes interference and it makes hard to decode the received symbol at a destination and makes error performance degradation. Also, the proposed scheme obtains spatial diversity gain compared to the conventional FIC. Finally, the proposed scheme has the same BER performance as ML because low-complexity QRD-M has optimal error performance.

Figure 4 also shows BER performances with respect to SNR for the conventional FIC and the proposed scheme. The main difference compared to Figure 3 is that the distances from one relay to another relay are normalized to $0.1,0.3,0.6$ and 0.9. The BER performance for the conventional FIC is degraded with respect to decreased distance because the power for interference symbol is increased when the distance between relays is decreased and it makes severe error propagation at a destination. However, the BER performance for the proposed scheme is increased with respect to decreased distance. At a relay, the low-complexity QRD- $M$ detects the received symbol in order of higher post SNR and cancels it from the received symbol. That is, the interference symbol is previously cancelled from the received symbol and desired symbol is cancelled later because the power for interference symbol is higher than the power for desired symbol when the distance rate is lower than 1. After the cancellation of the interference symbol, post SNR for the system is increased and it leads to increase of the error performance with respect to decreased distance. Also, the rate of degradation for the BER performance of the proposed scheme is lower than the conventional FIC because the 
proposed scheme obtains spatial diversity gain. Finally, the BER performance for the proposed scheme at distance rate 0.01 is slightly better than the distance rate 0.3 because the proposed scheme removes the IRI nearly perfectly at the distance rate 0.3 . And it has almost the same BER performance for the conventional CDD in one-path relay system which is not suffer from any IRI. Specifically, the BER performance for the proposed scheme approaches to the BER performance for the conventional CDD with respect to increased SNR. The distance rate 0.01 from one relay to another relay denotes that relays are very close to each other and it leads to remove the IRI perfectly at a relay when the SNR is high.

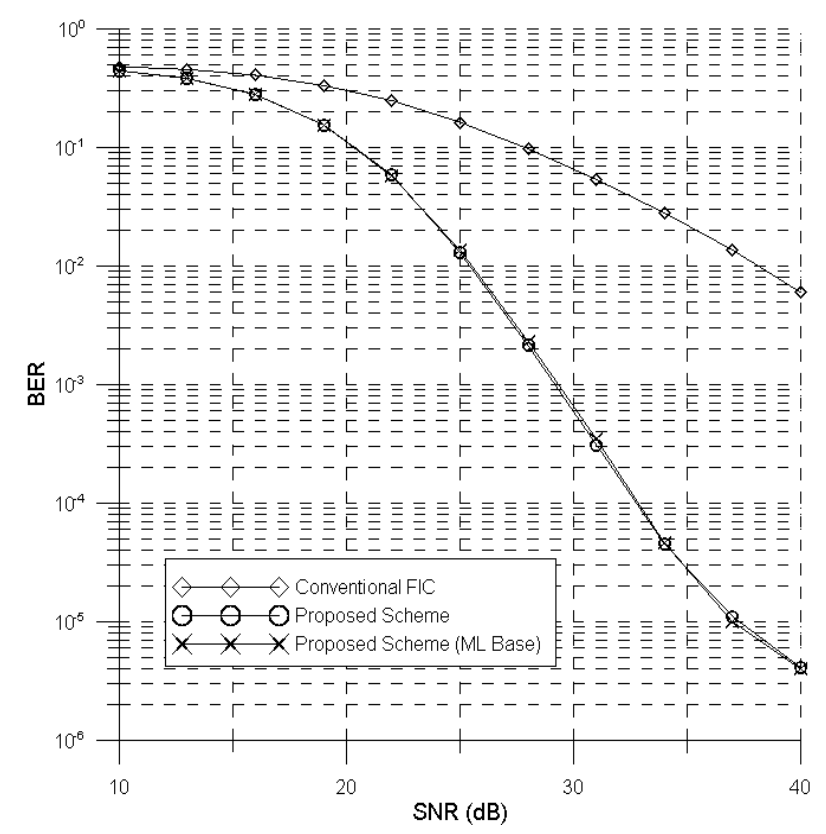

Figure 3. The BER performances for the conventional FIC and the proposed scheme. BER: bit error rate, FIC: full interference cancellation.

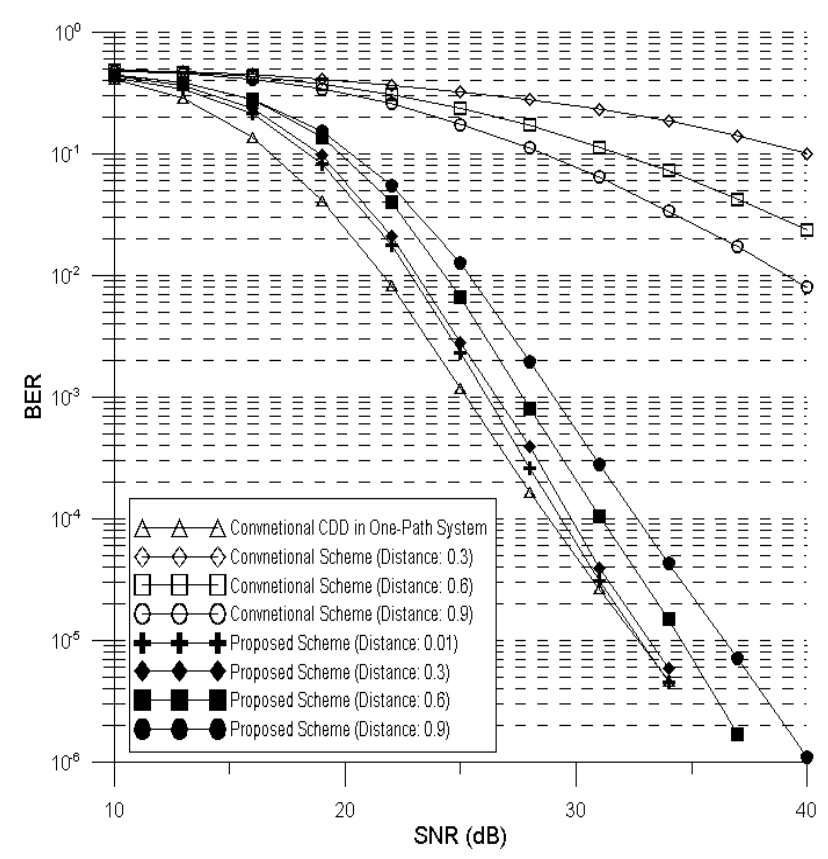

Figure 4. The BER performances for the conventional FIC and the proposed scheme. 
Figure 5 shows the required value of SNR to obtain targeting BER performance of $10^{-3}$ with respect to the distance from a source to a relay. The distances from a relay to a destination and one relay to another relay are normalized to 1 . The difference for the distance between the conventional FIC and the proposed scheme is more than 2.5 for certain required value of SNR. So, the proposed scheme has more than 2.5 times of transmission coverage compared to the conventional FIC.

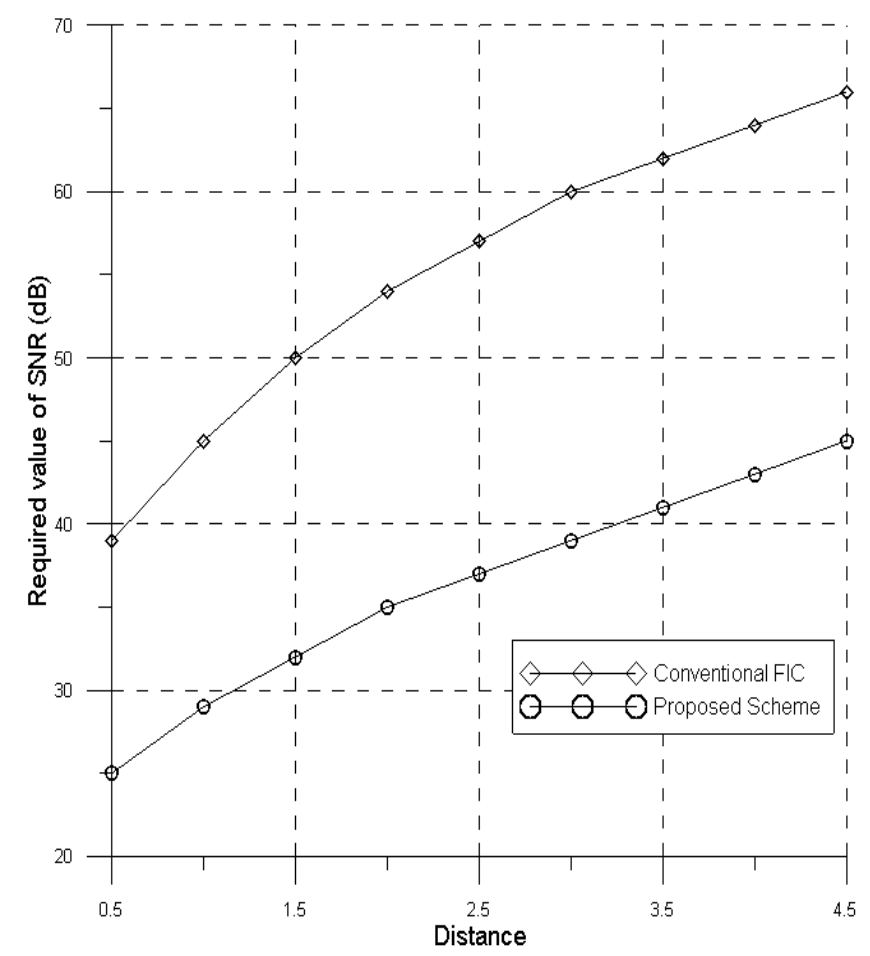

Figure 5. The required value of signal-to-noise ratio (SNR) to obtain targeting BER performance of $10^{-3}$.

Table 4 shows the complexity for the conventional FIC and the proposed scheme at a destination. The variable $N$ which is more than two is the number of used antennas. For comparison of the complexity, the number of complex additions, multiplications and divisions is shown. The proposed scheme does not require complex equalizer because interference symbol is removed at a relay unlike the conventional FIC which removes interference symbol at a destination. For more than three of $N$, the whole complexity for the proposed scheme is higher than the conventional FIC. However, the increase rate for the complexity of the proposed scheme is linearly increased with respect to the number of antennas and it is slightly large compared to the conventional FIC.

Table 4. The complexity at a destination for the conventional FIC and the proposed scheme.

\begin{tabular}{ccc}
\hline Scheme & Conventional FIC & Proposed Scheme \\
\hline Addition & 14 & $\begin{array}{c}4 N \\
(2 N \text { for (a) in Equation (15) and } 2 N \text { for }(b) \\
\text { in Equation (15)) }\end{array}$ \\
\hline Multiplication & 22 & $12 N$ \\
& 2 & $\begin{array}{c}(4 N \text { for composite channel, } 4 N \text { for (a) in } \\
\text { Equation (15), } 4 N \text { for (b) in Equation (15)) }\end{array}$ \\
\hline Division & $2 N$ for division in Equation (15) \\
\hline
\end{tabular}




\section{Conclusions}

In this article, a new interference cancellation using MIMO is proposed in two-path relay systems for higher error performance. Using the MIMO signal detection at a relay, the proposed scheme fully removes the IRI which is caused by simultaneous transmission of a source and one of the relay nodes. So, the IRI is not propagated to a destination and the BER performance is improved because less errors are propagated. After the removal of IRI, the proposed scheme can use the DF at the relays unlike the conventional FIC. Also, the BER performance is not degraded when the distance from one relay to another relay is decreased. Therefore, the proposed scheme is effective in the situation of wireless systems that the mobile users operating as relay nodes move and approach each other. Also, unlike the original two-path relay system, the proposed scheme can obtain spatial diversity gain by constructing the new symbol structures and can be used well such as area for cell edge or environments for severe multi-path fading in wireless communication systems.

Acknowledgments: This work was supported by Institute for Information \& communications Technology Promotion (IITP) grant funded by the Korea government(MSIT) (No. 2017-0-00217, Development of Immersive Signage Based on Variable Transparency and Multiple Layers).

Author Contributions: J.-H.R proposed an algorithms for removal of IRI at the relays and low-complexity MIMO signal detection scheme; S.-S.P. advised an algorithm for higher error performance; H.-K.S. gave feedbacks about a modified algorithm and all simulation results. Also, H.-K.S. provided the experimental materials for better computational simulations and revised critical errors of the manuscript.

Conflicts of Interest: The authors declare no conflict of interest.

\section{References}

1. Saxena, R.; Hem Dutt, J. OFDM and Its Major Concerns: A Study with Way Out. IETE J. Educ. 2013, 8, $26-49$. [CrossRef]

2. Hwang, T.; Yang, C.; Wu, G.; Li, S.; Li, G.Y. OFDM and Its Wireless Applications: A Survey. IEEE Trans. Veh. Technol. 2009, 58, 1673-1694. [CrossRef]

3. Nosratinia, A.; Hunter, T.E.; Hedayat, A. Cooperative Communication in Wireless Networks. IEEE Commun. Mag. 2004, 42, 74-80. [CrossRef]

4. Su, W.; Sadek, A.K.; Liu, K.R. Cooperative Communication Protocols in Wireless Networks: Performance Analysis and Optimum Power Allocation. Wirel. Pers. Commun. 2008, 44, 181-217. [CrossRef]

5. Laneman, J.N.; Tse, D.N.; Wornell, G.W. Cooperative Diversity in Wireless Networks: Efficient Protocols and Outage Behavior. IEEE Trans. Inf. Theory 2004, 50, 3062-3080. [CrossRef]

6. Luo, C.; Gong, Y.; Zheng, F. Full Interference Cancellation for Two-Path Relay Cooperative Networks. IEEE Trans. Veh. Technol. 2011, 60, 343-347. [CrossRef]

7. Rui, X.; Wang, M.; Liu, S. Inter-Relay Interference Suppression for Two-Path MIMO Relay Systems. Electron. Lett. 2016, 52, 774-776. [CrossRef]

8. Ji, Y.; Han, C.; Wang, A.; Shi, H. Partial Inter-Relay Interference Cancellation in Two Path Successive Relay Network. IEEE Commun. Lett. 2014, 18, 451-454. [CrossRef]

9. Wolniansky, P.W.; Foschini, G.J.; Golden, G.D.; Valenzuela, R.A. V-BLAST: An Architecture for Realizing Very High Data Rates over the Rich-Scattering Wireless Channel. In Proceedings of the 1998 URSI International Symposium on Signals, Systems, and Electronics, Pisa, Italy, 2 October 1998; pp. 295-300.

10. Golden, G.D.; Foschini, C.J.; Valenzuela, R.A.; Wolniansky, P.W. Detection Algorithm and Initial Laboratory Results Using V-BLAST Space-Time Communication Architecture. Electron. Lett. 1999, 35, 14-16. [CrossRef]

11. Bohnke, R.; Wubben, D.; Kuhn, V.; Kammeyer, K.D. Reduced Complexity MMSE Detection for BLAST Architectures. In Proceedings of the Global Telecommunications Conference, San Francisco, CA, USA, 1-5 December 2003; pp. 2258-2262.

12. Wubben, D.; Bohnke, R.; Kuhn, V.; Kammeyer, K.D. MMSE Extension of V-BLAST Based on Sorted QR Decomposition. In Proceedings of the IEEE 58th Vehicular Technology Conference, Orlando, FL, USA, 6-9 October 2003; pp. 508-512.

13. Ro, J.H.; Kim, J.K.; You, Y.H.; Song, H.K. Low-Complexity QRD-M with Path Eliminations in MIMO-OFDM Systems. Appl. Sci. 2017, 7, 1206. [CrossRef] 
14. Kim, J.K.; Ro, J.H.; Song, H.K. A Simplified QRD-M Algorithm in MIMO-OFDM Systems. IEICE Trans. Fundam. Electron. Commun. Comput. Sci. 2017, 100, 2195-2199. [CrossRef]

15. Kim, J.K.; Choi, S.J.; Ro, J.H.; Song, H.K. Adaptive K-Best BFTS Signal Detection Algorithm Based on the Channel Condition for MIMO-OFDM Signal Detector. IEICE Trans. Fundam. Electron. Commun. Comput. Sci. 2017, 100, 2207-2211. [CrossRef]

16. Kim, K.J.; Yue, J.; Iltis, R.A.; Gibson, J.D. A QRD-M/Kalman Filter-Based Detection and Channel Estimation Algorithm for MIMO-OFDM Systems. IEEE Trans. Wirel. Commun. 2005, 4, 710-721. [CrossRef]

17. Lim, H.; Jang, Y.; Li, T.; Yoon, D. Improved QRD-M Algorithm Based on Adaptive Threshold for MIMO Systems. In Proceedings of the 2014 Sixth International Conference on Communication Systems and Networks (COMSNETS), Bangalore, India, 6-10 January 2014.

18. Sun, S.; Dai, Y.; Lei, Z.; Kenichi, H.; Kawai, H. Pseudo-Inverse MMSE Based QRD-M Algorithm for MIMO OFDM. In Proceedings of the IEEE 63rd Vehicular Technology Conference, Melbourne, Australia, 7-10 May 2006; pp. 1545-1549.

19. Bossert, M.; Huebner, A.; Schuehlein, F.; Haas, H.; Costa, E. On Cyclic Delay Diversity in OFDM Based Transmission Schemes. In Proceedings of the 7th International OFDM Workshop, Hamburg, Germany, 10-11 September 2002.

20. Alamouti, S.M. A Simple Transmit Diversity Technique for Wireless Communications. IEEE J. Sel. Areas Commun. 1998, 16, 1451-1458. [CrossRef]

(C) 2018 by the authors. Licensee MDPI, Basel, Switzerland. This article is an open access article distributed under the terms and conditions of the Creative Commons Attribution (CC BY) license (http://creativecommons.org/licenses/by/4.0/). 